\title{
Abstracts for the 15th Interim Meeting of the Australasian Society of Cytogeneticists Richmond, Australia, August 7-9, 2009
}

\author{
ALTERNATIVE TESTING METHODS AND \\ PALLISTER-KILLIAN SYNDROME \\ S. Bain', M. Digenis', R. Hutchinson', J. Nicholl', S. Smith' L. Teicher' W.Waters' \\ and E.Thompson ${ }^{2}$ \\ Cytogenetics Unit, SA Pathology, Women's and Children's Hospital, North Adelaide, \\ Australia \\ South Australian Clinical Genetics Service, SA Pathology, Women's and Children's \\ Hospital, North Adelaide, Australia
}

A female infant who delivered at 37 weeks gestation with dysmorphic features was referred for chromosome analysis. Birthweight was 4970 grams, length $53 \mathrm{cms}$ and head circumference $38 \mathrm{cms}$ (all above 90th centile) GTL banding of cord blood at 550 band resolution showed a karyotype $46, X X$ (100 metaphases). At one week of age, a clinical consult was undertaken by a Clinical Geneticist. The facial appearance was immediately recognizable as typical of Pallister-Killian syndrome (PKS) with hypertelorism, upward slanting palpebral fissures, a flat nasal bridge, a short nose with anteverted nares, a long philtrum and posteriorly rotated ears. Initial parental resistance to invasive testing via skin biopsy led to consideration of other specimen sources and testing procedures. As a consequence of these studies permission was obtained for a skin biopsy. A stored frozen amniotic fluid specimen taken at 32 weeks for fetal lung maturity and the cord blood sample previously used for initial cytogenetic testing were retrieved. A fresh buccal scraping was also collected and with the above samples processed to standard protocols for FISH analysis. A FISH probe for the chromosome 12 centromere (Poseidon) was hybridized to the patient slides. An abnormal and mosaic pattern was observed for all tissue types with 3 copies of the chromosome 12 centromere found in an overall average of $35 \%$. The lowest percentage was found in cord blood and the highest in amniotic fluid. Considering the recent publication that describes array CGH in PKS patients (Theisen et al. 2009), the WCH laboratory has undertaken its own evaluation with the Agilent $8 \times 60 \mathrm{~K}$ array to explore the use of new methodologies with syndromes previously described as exhibiting tissue restricted mosaicism. Results would indicate that molecular cytogenetic techniques can now accurately diagnose PKS syndrome and possibly other syndromes that were previously not detectable in PHA stimulated blood cultures.

\section{IDENTIFICATION OF NOVEL CO-LOCATING}

MICRODELETION AND MICRODUPLICATION SYNDROMES

IN 17P13.3 DISTINCT FROM ISOLATED LISSENCEPHALY SEQUENCE AND MILLER-DIEKER SYNDROME

D. L. Bruno 1.2,* B.-M.Anderlid ${ }^{3 *}$,A. Lindstrand ${ }^{3}$, C. van Ravenswaaij-Arts ${ }^{4}$, D. Ganesamoorthy', , J. Lundin', C. L. Martin ${ }^{5}$, J. Douglas 6 , C. Nowak ${ }^{6}$, M. P.Adam ${ }^{5}$, R. F. Kooy ${ }^{7}$, N.Van der $\mathrm{Aa}^{7}$, E. Reyniers ${ }^{7}$, G.Vandeweyer ${ }^{7}$, I. Stolte-Dijkstra ${ }^{4}$, T. Dijkhuizen ${ }^{4}$, A. Yeung', M. Delatycki', B. Borgström ${ }^{8}$, L. Thelin', C. Cardoso ${ }^{10}$ B. van Bon"', R. Pfundt" , B. B.A. de Vries"', D. J.Amor', P. James', H. R. Slater ${ }^{1,2}$ and J. Schoumans ${ }^{3}$

Victorian Clinical Genetics Service, Murdoch Childrens Research Institute, Melbourne, Australia

Department of Paediatrics, University of Melbourne, Royal Children's Hospital, Melbourne, Australia

Clinical Genetics Unit, Department of Molecular Medicine and Surgery, Karolinska Institutet, Stockholm, Sweden

Department of Genetics, University Medical Center Groningen, University of Groningen, The Netherlands

Emory University, Department of Human Genetics, Atlanta, United States of America

The National Birth Defects Center, Waltham, United States of America

University and University Hospital Antwerp, Antwerp, Belgium

Department of Endocrinology, Clinic of Pediatrics, Karolinska University Hospital, Huddinge, Sweden

Sachs' Childrens Hospital, Södersjukhuset, Stockholm, Sweden

INMED, INSERM U901, Université de la Méditerranee, Campus de Luminy, Marseille, France

"Department of Human Genetics, Radboud University, Nijmegen Medical Centre, Nijmegen, The Netherlands

These authors contributed equally to this work.

E-mail:damien.bruno@mcri.edu.au

Chromosome $17 \mathrm{p} 13.3$ contains extensive repetitive sequences and is a recognized region of genomic instability. Within this genomic region, haploinsufficiency of PAFAH1B1 (encoding LIS1) causes either isolated lissencephaly sequence or Miller Dieker syndrome, depending on the size of the deletion. We have identified a more distally located and previously unknown microdeletion syndrome mapping to $17 \mathrm{p} 13.3$ in eight unrelated individuals as well as overlapping microduplications in four unrelated individuals. Detailed molecular analyses of the $17 \mathrm{p} 13.3$ microdeletions identified a minimal region of overlap (MRO) spanning $140 \mathrm{~kb}$ and encompassing four genes, including one likely candidate of clinical significance ( $C R K$ encoding Crk). In seven out of eight deletions an additional candidate gene, $Y W H A E$ encoding 14-3-3e, was included in the deletion. The clinical features of individuals with microdeletions included growth retardation, facial dysmorphism and developmental delay, but notably did not include lissencephaly. In contrast, the co-locating microduplications with a minimal MRO spanning $191 \mathrm{~kb}$ encompassing two genes, including one likely candidate of clinical significance (YWHAE encoding 14-3-3 $\varepsilon)$, were associated with autism, motor delay and subtle dysmorphism. We show that these genomic rearrangements are not associated ith low copy repeats (LCRs) and probably occurred by diverse molecular mechanisms.

\section{CYTOGENETICS IN THE MANAGEMENT OF MYELOID MALIGNANCIES}

\section{J. Campbell}

Victorian Cancer Cytogenetics Service, St Vincent's Hospital, Melbourne, Australia

\section{E-mail: Lynda.campbell@svhm.org.au}

Cytogenetics is an integral part of the management of patients with acute myeloid leukemia (AML) and myelodysplastic syndromes (MDS). Chromosome abnormalities are observed in approximately $60 \%$ of de novo cases of AML and the 2008 WHO classification considerably extended the list of AML subtypes defined by a specific genetic alteration. It is therefore no longer possible for a hematologist to categorize an AML without the cytogenetic analysis and this, together with increasingly tailored therapy regimens, places significant pressure on the cytogenetics laboratory. MDS is a more chronic disorder with fewer cases showing an abnormal karyotype and only one specific subtype defined by a chromosome abnormality - MDS with isolated $\operatorname{del}(5 \mathrm{q})$. In the past, the only appropriate care for MDS patients was supportive. However, there are now a number of drugs available with activity in MDS and the prognostic stratification of MDS has become an important part of management. The International Prognostic Scoring System (IPSS) divides chromosome abnormalities in MDS into three groups; good, intermediate and poor. This classification has been in use for the last decade but a recent study of $>2,500$ MDS cases introduced a four tier classification with a number of extra abnormalities moved into the good prognosis group. This classification also divided complex karyotypes into those with 3 abnormalities (Intermediate-2) and those with $>3$ (Poor prognosis). Such a precise definition of a complex karyotype presumes that all chromosome abnormalities are counted according to the same guidelines. The scoring of chromosome abnormalities and assignment of the corresponding IPSS score in complex karyotypes was assessed recently and identified major difficulties achieving concordance between cytogeneticists and hematologists. There is immediate need for an international standardized complexity scoring system and cytogeneticists should become more proactive in the management of MDS patients by including the IPSS karyotype score on the cytogenetics reports of all newly diagnosed MDS patients. 
CYTOGENETIC AND MOLECULAR CHARACTERIZATION OF A 7021.31 MICRODELETION: A CASE STUDY OF COMPLEMENTARY TECHNOLOGIES

S. Daly', E. Doherty', F.Ashton', L. C.Williams², S.Aftimos ${ }^{3}$, D. R. Love ${ }^{1,2}$ and A. George'

${ }^{\prime}$ LabPLus, Auckland City Hospital, Auckland, New Zealand

${ }^{2}$ School of Biological Sciences, University of Auckland, Auckland, New Zealand

${ }^{3}$ Northern Regional Genetic Service, Auckland City Hospital, Auckland, New Zealand

\section{E-mail: SheridanD@adhb.govt.nz}

The 17q21.31 microdeletion syndrome encompasses clinical features that include a distinctive facial appearance with a characteristic tubular or pear-shaped nose, hypotonia, developmental delay, abnormal hair color or texture, cryptorchidism and a friendly disposition. We have characterized a patient with a $17 \mathrm{q} 21.31$ microdeletion that, together with data from other Australian laboratories, expands the phenotype. The analysis of the New Zealand patient encompassed conventional karyotyping, as well as high resolution BAC and oligonucleotide-based array analysis. We identified the at-risk chromosome for the interstitial deletion using haplotype analysis of parental and proband samples, which comprised a panel of three microsatellites and a microdeletion in an intron of the MAPT gene. The precise location of the breakpoint was deduced by undertaking long range PCR of the proband's DNA using a number of oligonucleotides flanking the deduced deleted region. Finally, prenatal analysis of a further pregnancy of the proband's parents was undertaken using MLPA in order to exclude the presence of the deletion event.

\section{DEVELOPMENT OF A MULTIPLEX LIGATION-DEPENDENT PROBE AMPLIFICATION ASSAY FOR DIAGNOSIS AND ESTIMATION OF THE FREQUENCY OF SPINOCEREBELLAR ATAXIA TYPE 15}

D. Ganesamoorthy', D. L. Bruno', J. Schoumans ${ }^{2}$, E. Storey ${ }^{3}$, M. B. Delatycki ${ }^{4}$, D. Zhu ${ }^{5}$, M. K.Wei ${ }^{5}$, G. A. Nicholson ${ }^{5}$, R. J. M. Gardner ${ }^{4}$, and H. R. Slater ${ }^{1,4}$ 'VCGS Pathology and Murdoch Children's Research Institute, University of Melbourne, Department of Paediatrics, Royal Children's Hospital, Parkville, Australia

${ }^{2}$ Department of Molecular Medicine and Surgery, Karolinska Institute, Karolinska University Hospital Solna, Stockholm, Sweden

${ }^{3}$ Department of Medicine, Alfred Hospital, Monash University, Melbourne, Australia ${ }^{4}$ Genetic Health Services Victoria, Melbourne, Australia

${ }^{5}$ ANZAC Research Institute, University of Sydney, Department of Medicine, Concord Hospital, Sydney, Australia

\section{E-mail: howard.slater@ghsv.org.au}

Spinocerebellar ataxia type 15 (SCA15) is a slowly progressive neurodegenerative disorder characterized by cerebellar ataxia. Mutation of the ITPR1 gene (inositol 1,4,5-triphosphate receptor, type 1) has been identified recently as the underlying cause, and in most cases the molecular defect is a multiexon deletion. To date, 5 different SCA15 families have been identified with ITPRI gene deletion. We have designed a synthetic, dual-color multiplex ligation-dependent probe amplification (MLPA) assay that measures copy number with high precision in selected exons across the entire length of ITPRI and the proximal region of the neighboring gene, SUMF1 (sulfatase modifying factor 1). We screened 189 idiopathic ataxic patients with this MLPA assay and identified ITPRI deletion of exons $1-10$ in the previously reported AUS1 family ( 4 members) and deletion of exons $1-38$ in a new family ( 2 members). In addition to the multiexon deletions, apparent single-exon deletions identified in 2 other patients were subsequently shown to be due to single-nucleotide changes at the ligation sites. The frequency of ITPR 1 deletions is $2.7 \%$ in known familial cases. This finding suggests that SCA15 is one of the 'less common' SCAs. Although the deletions in the 5 families identified worldwide thus far have been of differing sizes, all share deletion of exons 1-10. This region may be important, both in terms of the underlying pathogenetic mechanism and as a pragmatic target for an accurate, robust, and cost-effective diagnostic analysis.

\section{NOVEL EPIGENETIC MARKERS OF THE FRAGILE X ALLELES}

D. E. Godler', F. Tassone ${ }^{2}$, H. R. Slater ${ }^{3}$, D. Z. Loesch', F. Gehling', T. Burgess ${ }^{3}$,

D. Ganesamoorthy ${ }^{3}$, L. Gordon 5 ,A. Evins ${ }^{6,7}$, and A. K. H. Choo'

'Chromosome and Chromatin Research Laboratory, The Murdoch Childrens Research Institute, Department of Paediatrics, University of Melbourne, Royal Children's Hospital, Melbourne, Australia

2 Department of Biological Chemistry, School of Medicine, University of California Davis, Sacramento, United States of America

3 VCGS Cytogenetics Laboratory, Murdoch Childrens Research Institute, Royal Children's Hospital, Melbourne, Australia

${ }^{4}$ The Olga Tennison Centre for Autism Research, School of Psychological Science, La Trobe University, Melbourne, Australia

${ }^{5}$ The Bioinformatics Unit, Murdoch Childrens Research Institute, Department of Paediatrics, University of Melbourne, Royal Children's Hospital

${ }^{6}$ Department of Neurology, Royal Melbourne Hospital, Melbourne, Australia

${ }^{7}$ Department of Medicine, University of Melbourne, Melbourne, Australia

E-mail: david.godler@mcri.edu.au

We have identified a number of novel epigenetic markers for Fragile X Syndrome (FXS), based on the methylation profiles of regions adjacent to the FMR1 CpG island, using MALDI-TOF MS. We named the most promising region Fragile X Related Epigenetic Element 1 (FREE1). Our pilot sample consisted of DNA from 62 healthy controls and 41 FXS individuals, from whole blood, EBV transformed lymphoblasts and Chorionic Villi Samples. FREE1 methylation analysis was used to identify FXS methylated alleles with specificity of $>99 \%$ and sensitivity greater than $10 \%$ methylation. It closely reflected assessment of the FMR $1 \mathrm{CpG}$ island using Southern blot analysis, but required $~ 100$ fold less DNA quantity, was more rapid, and $\sim 10$ fold less expensive. FREE1 methylation pattern was generally consistent between the cell types and closely reflected adult pattern of X-inactivation. Notably, both the classical CpG island and the FREE1 region escaped methylation related to X-inactivation in 15 weeks' old CVS cells, suggesting that there may be a functional role for both regions in females during late foetal development. To elucidate the relationship between changes is FREE1 methylation and bi-directional transcription at the FMR1 locus, we have treated cell lines with 5-aza-2'deoxycytidine, and identified critical CpG units within the FREE1 region closely related to FMR1 and FMR4/ASFMR1 expression. These sites were co-localized with putative SRY and GATA1/2 binding regions. In conclusion, we have identified a novel epigenetic marker for FXS FREE1 using MALDI-TOF MS that may overcome the problems associated with screening for large methylated CGG expansions across different tissues. FREE1 methylation was closely related to that of the FMR1 CpG island, in adult and foetal tissues, and was linked to bi-directional transcription at the locus. Due to the high throughput nature of the assay, it may become an ideal tool for newborn or prenatal screening of FXS.

\section{T(X;18) IN A FAMILY WITH PREMATURE OVARIAN FAILURE AND MALE FERTILITY}

S. Grehan, N. Martin, and R.Watson

Queensland Fertility Group, Brisbane, Australia

E-mail: stephanieg@qfg.com.au

$\mathrm{X}$-autosome translocations in females present with a variable clinical phenotype, including miscarriage, gonadal dysfunction, expression of an $\mathrm{X}$-linked recessive disorder or congenital abnormalities with or without developmental delay. Gonadal dysfunction eg premature ovarian failure (POF) is a function of the breakpoints on the $\mathrm{X}$ chromosome. In females carriers skewed $X$ inactivation plays a role in maintenance of genetic balance, with the normal $X$ preferentially inactivated, to prevent inactivation of the autosomal material on the derivative X. Males are almost universally reported to present with infertility. The infertility is due to spermatogenic arrest, usually at pachytene of meiosis I. We present a sibship of 3 individuals where one female is a balanced carrier $(\mathrm{t}(\mathrm{X} ; 18)(\mathrm{q} 22.3 ; \mathrm{q} 23)$, one male a balanced carrier and one sister unbalanced with her karyoytpe 46,X,der(X)t(X;18)(q22.3;q23) resulting from adjacent 1 segregation. This translocation has been inherited from their mother. Both sisters presented with premature ovarian failure (POF) at ages 33 and 29 years. The mother also experienced premature ovarian failure, but had all 3 children in her early 20 s. The carrier brother and his partner have undergone three cycles of IVF with the birth of dizygotic male twins both with a normal 46,XY karyotype. His sperm count ranged from $5 \times 10^{6}$ to $20 \times 10^{6} / \mathrm{ml}$, necessitating ICSI with IVF. Female carriers of $\mathrm{X}$-autosome translocations should be encouraged to complete their families in their early 20 . Counseling for male carriers detected prenatally should make mention rare cases where fertility has been reported. With the advent of assisted reproductive technologies (ART) and the use of testicular biopsy for sperm retrieval, more male carriers of such translocations may be able to father children. 
INCREASING GAIN OF A 500KB REGION AT 20011.21

DURING DISEASE EVOLUTION IN A CASE OF ERYTHROLEUKEMIA SUPPORTS SELECTED AMPLIFICATION OF AN ONCOGENE IN THE AMPLIFIED REGION

\section{R. N. MacKinnon' and L. J. Campbell',}

Victorian Cancer Cytogenetics Service, St Vincent's Hospital, Melbourne, Australia Department of Medicine (St Vincent's Hospital), University of Melbourne, Australia

E-mail: ruth.mackinnon@svhm.org.au

Loss of $20 \mathrm{q} 12$ is a recurrent abnormality in myelodysplastic syndromes (MDS) and acute myeloid leukemia (AML). A 1.7Mb combined myeloid common deleted region (CDR) has been defined but a tumor suppressor gene in this region has not been definitively identified. In $40 \%$ of a series of 41 cases with unbalanced translocations and loss of $20 \mathrm{q} 12$, there was gain of the retained part of the deleted chromosome 20, suggesting that a gene on the retained part of the chromosome contributes to the oncogenic phenotype. We have identified a series of five cases with MDS or AML, with loss of the 20q12 CDR and localized amplification in 20q11.21. Using array $\mathrm{CGH}$ we identified a core $250 \mathrm{~kb}$ amplified segment containing four complete genes. In one of these cases karyotype evolution was studied using BAC FISH and array CGH, and showed increased gain of the $20 \mathrm{q} 11.2$ core amplified region over time. The 38 year-old male presented with erythroleukemia and a stemline consisting of a single unbalanced translocation, a dic $(17 ; 20)(\mathrm{p} 11.2 ; \mathrm{q} 11.2)$, together with other more complex derivative cell lines. At diagnosis there was an average gain of one copy of a $2 \mathrm{Mb}$ region of $20 \mathrm{q} 11.2$. Remission was achieved after allograft. At relapse new derivatives of the $\operatorname{dic}(17 ; 20)$ were found. A reduced $500 \mathrm{~kb}$ region was now present in five copies on average in the $70 \%$ abnormal cells. This pattern of karyotype evolution shows increasing amplification of a common $500 \mathrm{~kb}$ region which includes the $250 \mathrm{~kb}$ core amplified region, and is supportive evidence that one of the four genes in the core amplified region - TM9SF4, HCK, POFUT1 and PLAGL2 - is an oncogene active in MDS/AML. These five patients had a diagnosis of erythroleukemia (AML-M6) or MDS with erythoid hyperplasia and morphology approaching erythroleukemia. Our observations support a role for the acquisition of extra copies of the critical 20q11.2 region in the transformation of MDS with del(20q) to erythroleukemia.

\section{GENETICS OF MALE INFERTILITY: A CLINICAL PERSPECTIVE}

R. I. McLachlan

Prince Henry's Institute of Medical Research, Monash Medical Centre, Clayton and Monash IVF, Richmond, Australia

Male factor infertility is the sole or contributory reason for half of assisted reproductive treatments (ART) and intracytoplasmic sperm injection (ICSI) now provides fertility for many previously sterile men. Primary spermatogenic failure is a collective term for a heterogenous group of disorders that are manifest by abnormal sperm number, motility and/or structure/function. Most cases are unexplained but increasingly genetic factors are emerging. Their identification is essential in informing couples about the prospect for normal pregnancy, transmission of infertility and/or nongonadal disease in offspring. Sex chromosomal aneuploidy and autosomal translocations/inversions are found in $\sim 7 \%$ men with spermatogenic failure and may impact the efficacy and safety of ART. Klinefelter's syndrome is the commonest disorder but many men are otherwise healthy thus routine karyotyping is recommended for ICSI candidates with sperm densities < $10 \mathrm{million} / \mathrm{ml}$. A 3-fold higher prevalence of apparently de novo chromosomal anomalies in ICSI offspring of 46XY infertile men may be a result of unrecognized aneuploidy in their ejaculated/testicular sperm. Yq chromosome (micro)deletions result in los of key spermatogenic genes and are found in $\sim 4 \%$ men with sperm densities $<5$ million $/ \mathrm{ml}$. Routine testing is recommended but laboratories must use strict quality assurance procedures. Most deletions involve the AZFc region with severe oligozoospermia or the presence of testicular sperm and vertical transmission to male offspring via ICSI will result in later fertility defects. A smaller AZFc subdeletion, termed gr/gr, is compatible with natural fertility in some populations but is a risk factor for infertility in others. Bilateral congenital absence of the vas deferens (BCAV) carries an $85 \%$ chance of (compound) heterozygosity for CFTR mutations. Routine testing using an ethnically relevant panel is essential in classic BCAV or whenever there is evidence of variant clinical presentations. Couples must be aware of residual risk despite negative testing and conversely of the options for PGD if applicable. Other genetic associations/defects have not entered routine practice due to their rarity, or lack of predictive power or therapeutic implications. For example, understanding of the genetic regulation of puberty and hypogoandotropic hypogonadism has grown rapidly while androgen receptor mutations or CAG repeat polymorphisms in exon 1 are associated with infertility and androgen deficiency. Tests of sperm DNA quality shows some relationship to reproductive outcomes however currently this assessment lacks sufficient predictive power to effectively inform or modify clinical treatment pathways.

\section{OF MICE AND MEN: RAPID EVOLUTION OF MOUSE AND HUMAN Y CENTROMERES}

\section{D. Pertile ${ }^{1,2,3}$, K. H.A. Choo ${ }^{1,2}$, and P. Kalitsis ${ }^{1,2}$}

${ }^{\prime}$ Murdoch Childrens Research Institute, Melbourne, Australia

Department of Paediatrics, University of Melbourne, Melbourne, Australia

VCGS Pathology, Royal Children's Hospital, Melbourne, Australia

E-mail: mark.pertile@ghsv.org.au

The centromere is a specialized structure that plays a fundamental role in the faithful segregation and transmission of eukaryotic chromosomes during cell division. Although this function is highly conserved across all eukaryotes, the DNA sequence that underlies it is not, with unique centromere sequences found amongst all model multicellular eukaryotes including plants, flies, mice and primates. Common to all however, is a satellite sequence that is AT-rich and highly repetitive. This monotonous repetition makes centromeres resistant to current sequence assembly techniques, meaning they are excluded from genome assemblies despite their basic unit of repetition (called a monomer) being well documented. We have recently identified and characterized the mouse $\mathrm{Y}$ centromere sequence, which we found in a fully sequenced BAC clone containing the entire mouse $\mathrm{Y}$ centromere satellite array. This clone provides a unique opportunity to study the evolution and fine sequence structure of an entire endogenous mammalian centromere for the first time. Using bioinformatics, cytological and molecular genetic techniques, we demonstrate that the mouse Y centromere DNA has a higher-order repeat sequence organization very similar to human centromeres, but quite distinct from the molecular organization of other mouse centromeres, which lack this genomic sequence architecture. We have used comparative sequence analysis to show that the mouse Y centromere is undergoing an extremely rapid evolution, and that this accelerated evolution is also a feature of the human Y centromere.

\section{B-CELL ALL WITH TWO UNRELATED CYTOGENETIC \\ ABNORMALITIES}

S. Prabhu', S. Diaz', D. J. Gottlieb², W.Varikatt ${ }^{3}$, and A. Smith'

${ }^{1}$ Department of Cytogenetics, Children's Hospital at Westmead, Australia

${ }^{2}$ Department of Haematology, University of Sydney, Australia

${ }^{3}$ Department of Histopathology, Westmead Hospital, Australia

E-mail: sakship@chw.edu.au

The development of myelodysplastic syndrome (MDS) has rarely been observed in patients with acute B-lymphocytic leukemia (B-ALL). The discussion concerning the pathogenesis of the simultaneous occurrence of these two malignancies has been speculative - two separate abnormal clones or a common stem cell malignancy. We present a case of a 64-yearold woman who was evaluated for low platelet count and peripheral blasts. On the basis of the morphology, flow cytometry and the lack of myeloid associated markers, a diagnosis of precursor B-ALL was made. Cytogenetic analysis of the diagnostic bone marrow specimen revealed two unrelated abnormal clones with distinct morphologies. One clone had a dicentric $(7 ; 9)(\mathrm{p} 11 ; \mathrm{p} 11)$, resulting in the deletion of $7 \mathrm{p} \& 9 \mathrm{p}$. The other clone showed trisomy $8[+8]$. The $\operatorname{dic}(7 ; 9)$ is a rare but recurrent abnormality in B-cell ALL. Trisomy 8 as a sole abnormality is commonly associated with myeloid malignancies. This result could suggest a possible underlying MDS in this patient with B cell ALL. After standard treatment for B-cell ALL, BM cytogenetic analysis showed 3/40 cells with trisomy 8 . The result indicated residual disease for this cell line. Furthermore the poor response to treatment was inconsistent with the morphology report which indicated total remission. The unique aspect of this case is the observation of two unrelated clones in an individual suggestive of concomitant malignancies.

\section{COPY NUMBER VARIANT (CNV) ANALYSIS: FISHING FOR THE TRUTH AMID RED HERRINGS}

\section{S. Singh', E. Doherty', L. Williams ${ }^{2}$, S. Aftimos ${ }^{3}$, D. Love ${ }^{1,2}$, and A. George}

LabPLus, Auckland City Hospital, New Zealand

School of Biological Sciences, University of Auckland, New Zealand

Northern Regional Genetic Service, Auckland City Hospital, New Zealand

E-mail: shalinders@adhb.govt.nz

With the advent of microarrays we are presented with a technology that can identify whole genome copy number variants (CNVs). Critically, laboratory ethics drive us to confirm these variants using alternative analyses; however, the equal challenge is to assess array data for biological relevance. It is here that the scientist interfaces with dynamic databases that are not yet mature. Here we present a case referred with dysmorphic features and truncus arteriosis. Conventional karyotype analysis revealed a female karyotype with a de novo interstitial deletion within Xq27. We undertook further analysis of this deletion using a combination of microsatellite analysis and real-time PCR that identified the lack of inheritance of the paternal allele. Finally, we interrogated the patient's genomic 
DNA using high density microarray (Affymetrix SNP 6.0 chip) analysis, which identified a large CNV encompassing the FMR1 gene as well as a number of other CNVs. The location and extent of the FMR1 gene deletion did not correlate with the symptoms of our patient, nor did any CNV that the array data revealed. This case highlights the obvious point that arrays are just one tool in identifying the genetic underpinning of dysmorphism, and that accessible array databases such as DECIPHER require continuing clinical input in order that the scientist can guide the clinician in linking cause and effect.

\section{A THREE GENERATION FAMILY WITH DEL(14)(013.3021.3) WITH VARIABLE PHENOTYPIC EXPRESSION}

\section{P. Sutedja, D. Hung, M.Alcausin, and G. B. Peters \\ Department of Cytogenetics, Department of Clinical Genetics, The Children's Hospital at Westmead, Sydney, Australia}

E-mail: putras@chw.edu.au

Chromosome deletions of euchromatic segments usually produce an abnormal phenotype. We present a family of three generations with an interstitial deletion of chromosome 14q13.3q21.3 exhibiting variable phenotypic expression. A 27 year old female with hypodontia and imperforate anus was referred to our department for cytogenetic analysis. G-band analysis showed an abnormal female karyotype with an interstitial deletion on the long arm of chromosome 14: 46,XX, del(14)(q13q21). Pedigree and cytogenetics analysis showed the proband's father and two sisters also carried the same deletion and shared similar phenotype as the patient. In addition, one of the proband's sisters has variant Turner syndrome: 46,X,i(Xq)/45,X. The proband's mother has a normal karyotype. The proband's son has inherited the $14 \mathrm{q}$ deletion, although, he has a different phenotype to the rest of the family, with features including bilateral talipes and bilateral hip dislocation. Array CGH studies on the proband's son using the BlueGnome $4 \mathrm{~K}$ Cytochip confirmed the deletion and characterized it as having a minimum size of $7.8 \mathrm{Mb}$ and maximum size of $12.3 \mathrm{Mb}$. The minimum number of genes in the segment is 21 . The rest of the family was studied using the Agilent $44 \mathrm{~K}$ oligo platform, refining the size of the deleted segment to a minimum of $11.0 \mathrm{Mb}$ and a maximum of $11.3 \mathrm{Mb}$ (and 21 to 25 genes). The breakpoint of the deletion is also refined to bands 14q13.3 and $14 \mathrm{q} 21.3$. No innocuous copy number variants (CNVs) correspond to this large deletion. The deleted region is gene-poor compared to the genome average. This may contribute to the mild phenotype observed. Other factors to consider include incomplete penetrance, genomic imprinting, the unmasking of recessive allele on the non-deleted chromosome 14 or general loss of developmental canalization.

\section{MOLECULAR KARYOTYPING: FROM POSTNATAL}

\section{TO PREIMPLANTATION GENETIC DIAGNOSIS?}

\section{J. Rermeesch \\ Center for Human Genetics, K.U. Leuven, Belgium \\ E-mail: Joris.vermeesch@uzleuven.be}

Molecular karyotyping or genome wide array CGH has been implemented in postnatal diagnosis of patients with idiopathic mental retardation and congenital anomalies and is challenging conventional karyotyping as the prime diagnostic tool. Despite its successes, interpretation of the results coming from arrays with ever increasing resolution is becoming the main challenge. I will demonstrate how 'Mendelian copy number variants' apparently benign CNVs that can cause a disease phenotype dependent on copy number state, sex and genetic or environmental background require large scale collaborative efforts to collect sufficient data and the development of expert systems to provide accurate diagnosis. The technology has, more recently, been applied in a prenatal diagnostic setting. I will illustrate how the technology helps prenatal diagnosis, but also demonstrate the potential risks of using this technology. Finally, we developed a novel tool to genome wide screen CNV and SNP-genotype single cells. When applied to cleavage stage embryos from young fertile couples we discovered, unexpectedly, an extremely high incidence of chromosomal instability, a hallmark of tumorigenesis. Not only mosaicisms for whole chromosome aneuploidies and uniparental disomies but also frequent segmental deletions, duplications and amplifications that were reciprocal in sister blastomeres were detected in most cleavage stage embryos implying the occurrence of breakage-fusion-bridge cycles. As a consequence o, PGD-AS will not improve the selection of genetically normal embryos. This not only explains the low human fecundity but also identifies postzygotic chromosomal instability as a leading cause of constitutional chromosomal disorders.
THE CRADLE OF CONSTITUTIONAL CHROMOSOME REARRANGEMENTS IS THE CLEAVAGE STAGE EMBRYO

\section{J. R.Vermeesch}

Center for Human Genetics, K.U.Leuven, Belgium

E-mail: Joris.vermeesch@uzleuven.be

We recently developed a novel tool to genome wide screen $\mathrm{CNV}$ and SNP-genotype single cells (Vanneste et al., Nature Medicine, 2009). When applied to cleavage stage embryos from young fertile couples we discovered, unexpectedly, an extremely high incidence of chromosomal instability, a hallmark of tumorigenesis. Not only mosaicisms for whole chromosome aneuploidies and uniparental disomies but also frequent segmental deletions, duplications and amplifications that were reciprocal in sister blastomeres were detected in most cleavage stage embryos implying the occurrence of breakage-fusion-bridge cycles. We demonstrate the existence of those rearrangements in interphase nuclei. The type of rearrangements observed can likely explain the majority of constitutional rearrangements seen in miscarriages as well as live births such as deletions, duplications, inverted deletions duplications, ring chromosomes and mosaicisms of all of those rearrangements.

\section{REDUCED ANALYSIS TIME AND INCREASED CASE LOAD PER WEEK FOLLOWING IMPLEMENTATION OF THE} GSL-120 CYTOVISION SLIDE SCANNER

\section{D.Wright and A. Polihronis}

Sydney Genetics, Sydney, Australia

E-mail:dale.wright@sydneyivf.com

Automated slide scanners have promised improved efficiency and productivity gains for many years, however until recently appeared to have gained little acceptance possibly because of their limited slide capacity and generally a requirement for manually assisted x100 oil objective image capture. Capital expenditure is also a substantial consideration but it can be balanced against additional on-going increasing yearly salary costs. We introduced the GSL-120 with CytoVision (v4.01) (Genetix) in September ' 08 and predominantly have applied it to blood chromosome preparations. Conventional microscopy previously involved 10 cells counted, 5 cells analyzed band-for-band (550bphs), followed by capture of 2 cells ( $\leftrightarrow 100$ oil objective) with one karyotyped using CytoVision software. GSL-120 on-screen analysis of captured images $(\leftrightarrow 100$ oil objective) still involves 10 cells counted, 5 cells analyzed (550 bphs), however all 5 cells are also fully karyotyped. For our experienced analysts, on-screen analysis time has reduced to $\sim 45 \mathrm{~min}$ from $\sim 80 \mathrm{~min}$ for microscopy. Comparison of data pre- vs post implementation showed our overall sample numbers increased by 427 specimens while the number of analysts decreased slightly (9.6 vs. 9.2), case workload per analyst per week lifted from 10 to 12 cases, overall reporting time decreased slightly (12.9 vs. 11.9 days), and overtime hours decreased considerably ( 528 vs $41 \mathrm{hrs})$. The reduction in analysis time $(\sim 44 \%)$ and increased case workload per scientist is mainly a result of reduced time spent manually searching for suitable cells for analysis and capture. We have substantially reduced staff overtime and there are indications we may make further moderate productivity gains following integration of the GSL-120 with our laboratory LIMS.

\section{CYTOPLASMIC IMMUNOGLOBULIN LABELING WITH FISH: IMPROVED ABNORMALITY DETECTION RATES FOR PLASMA CELL MYELOMA}

A. Zordan, B. Mercer, C. Batzios, K. Rayeroux, and L. J. Campbell

Victorian Cancer Cytogenetics Service, St Vincent's Hospital, Melbourne, Australia

E-mail: Adrian.zordan@svhm.org.au

Cytogenetic studies of plasma cell myeloma (PCM) have routinely been hampered by the fact that terminally differentiated plasma cells do not proliferate well in vitro. Although FISH on interphase cells has the potential to circumvent this issue, it may not be sensitive enough to detect abnormalities in samples with low neoplastic cell counts. With increasing evidence showing the clinical and prognostic significance of genetic abnormalities in myeloma, these shortcomings can no longer be overlooked. Various strategies have been suggested for targeting FISH more specifically at plasma cells, ranging from plasma cell purification to combining the immunofluorescent labeling of plasma cells with FISH (cIg FISH). At the Victorian Cancer Cytogenetics Service, we have developed a protocol which allows for the positive identification of plasma cells by fluorescent labeling of cytoplasmic immunoglobulin $\kappa$ or $\lambda$ light chains. Combined with simultaneous interphase FISH, this 
approach has dramatically increased our abnormality detection rate and we have incorporated this method into our routine Myeloma FISH assays. The 3 assay, 6 probe FISH panel, which we used on our PCM patient samples, consisted of FGFR3/IGH (DF), D13S319/LAMP1 (DC) and TP53/D17Z1 (DC). Since 2007 we have processed 160 PCM samples for FISH, 30 of them using the cIg FISH approach. Our abnormality detection rate using interphase FISH alone was $34 \%$, but since the implementation of cIg FISH that figure has risen to $83 \%$. Given the relative ease with which our cIg FISH protocol can be applied to fixed cytogenetic suspensions (both prospectively and retrospectively) and the unequivocal improvement in the reliability of our results, we support recommendations being made worldwide that FISH in PCM should not be performed without some form of plasma cell identification or enrichment. 